\title{
Breakfast intake is associated with nutritional status, Mediterranean diet adherence, serum iron and fasting glucose: the CYFamilies study
}

\author{
Chrystalleni Lazarou ${ }^{1, *}$ and Antonia-Leda Matalas ${ }^{2}$ \\ ${ }^{1}$ Cyprus Food and Nutrition Museum, 5 Christianas Christofidou Street, 2417 Ekgomi Nicosia, Cyprus: \\ ${ }^{2}$ Department of Dietetics and Nutrition, Harokopio University, Athens, Greece
}

Submitted 26 August 2013: Final revision received 10 July 2014: Accepted 11 August 2014: First published online 7 0ctober 2014

\begin{abstract}
Objective: To assess the association of breakfast intake with Mediterranean diet adherence, physical activity levels, obesity, selected cardiovascular risk markers and Fe status.

Design: Cross-sectional study. BMI, body fat percentage and waist circumference were assessed. Physical activity was assessed using a pedometer and diet quality was evaluated by applying the KIDMED index. Blood tests to assess blood lipids, high-sensitivity C-reactive protein, fasting glucose, serum Fe and ferritin, as well as $\mathrm{Hb}$, were performed.

Setting: Primary-school children of the Troodos mountainous area in Cyprus.

Subjects: Eighty-three Cypriot children (mean age 9.2 (SD 1.7) years).

Results: Compared with breakfast skippers, regular breakfast eaters were $40 \%$ more likely to have a KIDMED score higher by one point on average $(\mathrm{OR}=1.41$; $95 \%$ CI 1.08, 1.84) after accounting for obesity levels and other confounders. Breakfast skippers, on the other hand, were by about $14 \%$ more likely to have a body fat percentage value higher by one unit, as well as higher values for both BMI and waist circumference. The relationship was significantly strengthened when combining body fat percentage and waist circumference into a composite variable $(\mathrm{OR}=0 \cdot 20 ; 95 \% \mathrm{CI}$ 0.06, 0.69). Fasting glucose was inversely correlated to breakfast intake in descriptive analyses, whereas serum Fe was positively correlated to breakfast intake after considering age, gender and diet quality. The latter relationship disappeared, however, after considering physical activity levels. Conclusions: Cypriot children who take breakfast regularly exhibit a higher adherence to the Mediterranean diet and have higher serum Fe concentrations and lower BMI, waist circumference, body fat percentage and fasting glucose levels, compared with children who skip breakfast. Public health professionals, educators and parents should prioritize on actions that will motivate children to regularly eat breakfast.
\end{abstract}

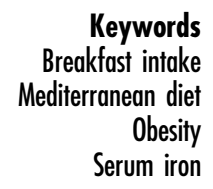

Breakfast intake is considered an essential characteristic of a healthy diet pattern for adults and children, and has been found to correlate with attributes of a healthy lifestyle such as physical activity levels, cardiovascular health indices as well as cognitive and academic performance in youth ${ }^{(1-3)}$. With regard to the association of breakfast with diet quality in children and adolescents, it has been shown that those who regularly eat breakfast have a diet of higher overall quality ${ }^{(4,5)}$, better food choices, regular eating patterns ${ }^{(6-8)}$, consistent energy intake ${ }^{(6)}$, and nutrient and fibre intakes that are more likely to meet the recommended values ${ }^{(2,9)}$. Concomitantly, skipping breakfast in children and adolescents has been associated with low overall diet quality $^{(10,11)}$, inability to reach recommended intakes of micronutrients ${ }^{(9,12-15)}$, higher energy intake at dinner ${ }^{(16)}$, lower intakes of healthful foods ${ }^{(17)}$ and increased intakes of low-nutritive-value foods ${ }^{(9,18)}$, such as high-fat snacks, later in the day ${ }^{(19)}$. Apart from the favourable associations with diet quality, regular breakfast intake has been associated in children with lower obesity rates (as assessed by $\mathrm{BMI})^{(9,20,21)}$, whereas breakfast skipping is associated with increased $\mathrm{BMI}^{(2,9,22,23)}$ and may be predictive of increased BMI in early adulthood ${ }^{(24)}$ even though skippers report lower energy intakes.

In view of the above data, the Expert Committee on Pediatric Obesity of the American Pediatric Academy 
included the daily intake of breakfast in the five most important behavioural recommendations aiming at the prevention of obesity ${ }^{(25)}$. The 2010 Dietary Guidelines for Americans also recommend regular intake of nutrient-dense breakfast for children and adolescents to maintain healthy weight, prevent obesity and improve nutrient intakes ${ }^{(9)}$. A number of public initiatives, such as US Department of Agriculture's School Breakfast Program ${ }^{(26)}$, the Primary School Free Breakfast Initiative in Wales ${ }^{(27)}$ and the European Union's Breakfast is Best campaign ${ }^{(28)}$, also promote regular breakfast intake.

Breakfast has been an indispensable component of past diets followed in Mediterranean societies, same as in many other societies in the Western world ${ }^{(29)}$. The study of traditional food practices in Cyprus shows that breakfast has been an essential element of Cypriots' daily routine ${ }^{(30)}$. Published data on the Cypriot population's breakfast habits are scarce and, to date, no systematic work has been attempted to investigate the association of breakfast intake with lifestyle and health indices. However, recent data suggest that Cypriots have abandoned this healthy habit: in the CYFamilies (Cyprus Families) study ( $n$ 83, mean age 9.2 (SD 1.7) years), daily breakfast intake was reported by $72 \%$ of the children and consumption of breakfast cereals by $55 \%$ of the children ${ }^{(31)}$; while in the nationwide CYKIDS (Cyprus Kids) study ( $n$ 1140, mean age 10.7 (SD 0.98 ) years), approximately $20 \%$ of boys and $25 \%$ of girls reported 'not having breakfast on most days of the week' ${ }^{\text {,(32) }}$ and $12 \%$ of all children had breakfast less than once per week ${ }^{(33)}$. In the same study, it was observed that a dietary pattern of increased consumption of milk and cereals, typical of regular breakfast eaters, was related to lower obesity levels but only in girls ${ }^{(34)}$. Thus, in view of the lack of data in this area, the aim of the present paper was to assess associations between breakfast intake and lifestyle attributes, i.e. diet quality and physical activity levels, as well as associations with Fe status and cardiovascular risk markers (i.e. obesity and serum cholesterol, TAG, fasting glucose and high-sensitivity C-reactive protein (hs-CRP)), in Cypriot children.

\section{Materials and methods}

\section{Study sample}

The CyFamilies study is a cross-sectional survey that was conducted during the 2006/07 school year among all primary-school students and their parents in the Troodos' mountainous villages in the Republic of Cyprus* aiming to evaluate anthropometric variables and dietary habits of the population. A total of 163 children aged 6-12 years were contacted and 160 agreed to participate (98.2\% participation rate). Those 160 children lived in five different villages, but since their schools were closed by the

\footnotetext{
* The Troodos district villages included Kyperounda, Xandria, Dymes,
} Agridia and Potamitissa.
Ministry of Education during previous years, they all attended one school when the study was conducted.

Dietary intake of the children and their parents was assessed using a semi-quantitative FFQ, consisting of 154 food items (including all common foods of the local Greek-Cypriot cuisine) and three supplementary questionnaires that evaluated other aspects of dietary habits (i.e. dietary beliefs, meal patterns). A detailed description of these questionnaires has been published previously ${ }^{(32)}$. For the present work, the KIDMED (Mediterranean Diet Quality Index for children and adolescents) questionnaire was also used to evaluate the children's adherence to the Mediterranean diet ${ }^{(35)}$. The KIDMED index (theoretical range -4 to 12 points), computed as detailed by SerraMajem et $a l .{ }^{(35)}$, is derived from sixteen components that summarize the principles of the Mediterranean diet prototype; a score of -4 to 3 points reflects a poor diet relative to the Mediterranean diet principles, whereas scores of 4-7 points and 8-12 points, respectively, signify average and good adherence to the principles of the Mediterranean diet. More specifically, the KIDMED index presumes a daily consumption of at least one serving of fruit and vegetables, while consumption of at least two servings of each group is preferable. The KIDMED index also assesses levels of consumption of dairy products (at least three servings daily are recommended), grains and cereals (recommended daily in breakfast, while cereal-based dishes should be consumed at least five times weekly), nuts, fish (weekly consumption of at least two to three servings is advisable) and olive oil. Dietary behaviours that are viewed as detrimental and alien to the principles of the Mediterranean diet are also assessed, i.e. frequent intake of sweets and candies (defined as more than twice daily), use of commercially baked goods and pastries for breakfast, consumption of fast foods and abstinence from breakfast. The KIDMED questionnaire has been validated against two $24 \mathrm{~h}$ recalls. Briefly, it was found that the KIDMED score followed increases in the intake of most of the sixteen vitamins and minerals examined. Higher scores were associated with decreased prevalence of inadequate intake $(<2 / 3$ of the Reference Nutrient Intake) for $\mathrm{Ca}, \mathrm{Mg}$, vitamin $\mathrm{B}_{6}$ and vitamin $\mathrm{C}$ in boys and girls ${ }^{(25)}$. For the present work, a modified KIDMED index (theoretical range -3 to 12 points) was used, after excluding the question on the regularity of breakfast intake, as this is the outcome to which all statistical evaluations were made. Dietary questionnaires were administered to pupils during class hours by a qualified nutritionist, following a standardized methodology for dietary data collection ${ }^{(31)}$; further details on study's methodology can be found elsewhere ${ }^{(31)}$.

Regular breakfast intake was assessed via a question asking whether children usually have breakfast on most days of the week. Breakfast was considered to be any food eaten and drunk before the commencement of school classes. On weekends, breakfast was considered to be 
anything eaten and drunk within two hours after rising in the morning. Examples of typical breakfasts were given, in order to help accurate recall. Having just a glass of milk or juice was considered a breakfast, whereas having only a cup of tea was not accepted as breakfast. Children who responded that they had breakfast on most days of the week were classified as 'breakfast eaters', whereas children who gave a negative response were classified as 'breakfast skippers'.

Physical activity levels in the children were estimated via the use of pedometers (DW-200; Yamax, Tokyo, Japan) following a specific protocol that has been described by other researchers ${ }^{(36)}$. Children wore the pedometer for at least two weekdays and one weekend day. Pedometers were not given to first grade students, as children of this age are not considered capable of accurately recording the number of counts on their pedometers.

Anthropometric data, i.e. weight, height and waist circumference (WC), were collected from children according to a standard protocol described by Heymsfield et al. ${ }^{(37)}$. Obesity was defined according to the International Obesity Task Force's age- and gender-specific BMI threshold criteria $^{(38)}$. Percentage of body fat (BF\%) was derived via bioelectrical impedance analysis, using a model BC-558 analyser (Tanita Corp., Tokyo, Japan), according to the protocol suggested by McCarthy et al. ${ }^{(39)}$. BF\% was categorized according to the reference values proposed by McCarthy et $a l^{(39)}$ for children by age and gender.

We combined the two variables of $\mathrm{WC}$ and $\mathrm{BF} \%$ into one categorical variable, named 'WC and BF\%', with two categories. First, WC and $\mathrm{BF} \%$ were each dichotomized as follows: for WC, $<77 \mathrm{~cm}$ ('normal') $v$. $\geq 77 \mathrm{~cm}$ ('above normal'), based on the 75th percentile threshold previously used in other studies among Cypriot children ${ }^{(40,41)}$; for $\mathrm{BF} \%$, under normal and healthy fat ('normal') $v$. overweight and obese ('above normal'), according to gender and age, as proposed by McCarthy et al. ${ }^{(39)}$. Children were classified in the 'lower risk' category if they had 'normal' values in both variables (WC and $\mathrm{BF} \%$ ) as defined above, whereas if they had at least one of the variables 'above normal' they were classified in the 'increased risk' category. Each 'lower risk' category was assigned a score of 0 , while a score of 1 was assigned to each 'increased risk' category; the possible score range for the 'WC and BF\%' variable was therefore 0 to 2. Having a higher score was an indicator of 'increased obesity risk'.

Biochemical examinations were performed by the state hospital of this district ${ }^{(31)}$. A total of eighty-three children underwent serum analyses following a $12 \mathrm{~h}$ overnight fast. All children were without clinical evidence of acute infection, dental problems and were not taking medications (i.e. corticosteroids) that could affect the levels of biochemical markers being measured. Blood was collected from the antecubital vein between 08.00 and 10.00 hours, the whole blood specimens were aliquoted into separate tubes and the collection tubes were immediately centrifuged at $3000 \mathrm{rpm}$ for 15 min (Eppendorf Centrifuge 5804) to obtain serum and plasma. Biochemical evaluations were performed by the same laboratory (Kyperounda State Hospital) in accordance with the criteria of the WHO Lipid Reference Laboratories. Haematological and biochemical measurements performed included whole blood cell count and serum $\mathrm{Hb}, \mathrm{Fe}$, ferritin, total cholesterol (TC), LDL cholesterol (LDL-C), HDL cholesterol (HDL-C), TAG, hs-CRP and fasting glucose levels. High-sensitivity CRP levels were assessed using particle-enhanced immune nephelometry in a model AU-400 automatic analyser (Olympus, Tokyo, Japan).

\section{Cardiovascular disease risk index}

Because descriptive univariate analyses failed to reveal any associations of blood lipids or hs-CRP with breakfast intake (Table 1), the synergistic effect of biochemical markers, i.e. blood lipids and hs-CRP, was examined by grouping them into one categorical variable, the 'cardiovascular disease risk index' (CDRI), to account for the magnitude of risk. Participating children were classified as having 'normal' biochemical indices using the American Heart Association's reference values for children ${ }^{(42)}$ : TC $<170 \mathrm{mg} / \mathrm{dl}$, HDL-C $>60 \mathrm{mg} / \mathrm{dl}$, LDL-C $<110 \mathrm{mg} / \mathrm{dl}$, TAG $<150 \mathrm{mg} / \mathrm{dl}$. The cut-off point for hs-CRP was set at $<0.2 \mathrm{mg} / \mathrm{dl}$, which represents average risk $^{(43)}$. Each 'normal' index was assigned a score of 0 , whereas a score of 1 was assigned to every 'above normal' index. Therefore, the possible score range of the CDRI was 0 to 5 . Having a higher score was an indicator of 'increased cardiovascular risk'. Children were classified in the 'lower risk' category if they had 'normal' values in all of the biochemical indices mentioned above, whereas if they had one index 'above normal', they were classified in the 'increased risk' category. Fasting glucose levels were not included in the CDRI, because, in descriptive analyses, they were found to be associated with breakfast intake, a fact that could falsely inflate the results of the CDRIbreakfast intake association to statistical significance.

\section{Bioethics}

The research design was approved by the Ministries of Education and Culture (Department of Primary Education) and Health, as Cypriot law provides for studies that are performed at school during formal school hours. The study was conducted according to the guidelines laid down in the Declaration of Helsinki and all procedures involving human subjects were approved by the Ministry of Education and Culture and the Ministry of Health. Written informed consent was obtained from the parent or guardian of each child prior to enrolment.

\section{Statistical analyses}

Continuous variables are presented as mean and standard deviation or as median and interquartile range, as appropriate, whereas categorical variables are presented as absolute and relative frequencies. Normality of the 
variables' distribution was evaluated using Q-Q plots and the Kolmogorov-Smirnov test. The hs-CRP variable was log transformed, as it was not normally distributed. Differences between the study groups (breakfast skippers, breakfast eaters) were tested by the independent $t$ test for normally distributed variables, by the Mann-Whitney $U$ test for variables with non-normal distribution or by the $\chi^{2}$ test for categorical variables. We further applied binary logistic regression analysis to evaluate the association between the regularity of breakfast intake (dependent outcome) and overall diet quality (as assessed by a modified KIDMED score), BMI, WC, BF\%, 'WC and BF\%' variable, fasting glucose, serum $\mathrm{Fe}$ and CDRI (independent variables) adjusting for potential confounders (age, gender and physical activity). All reported $P$ values are based on two-sided tests and the significance level was set to $0 \cdot 05$. The statistical software package IBM SPSS Statistics 18.0 for Windows was used for all statistical calculations.

\section{Results}

Selected demographic, anthropometric and lifestyle characteristics of the children by category of breakfast intake frequency are presented in Table 1. Significant differences were observed for overall diet quality (as assessed by the modified KIDMED score), fasting glucose levels and serum Fe levels. Specifically, KIDMED scores of breakfast skippers were, on average, one point lower compared with those of breakfast eaters. Further analyses (not shown here) showed that $14.0 \%$ of breakfast skippers had a 'poor diet quality' and $34.0 \%$ were classified in the 'good diet quality' group, whereas the corresponding values for breakfast eaters were $6.7 \%$ and $44.0 \%$, respectively.

With regard to serum $\mathrm{Fe}$, there was a difference of almost $13 \mu \mathrm{g} / \mathrm{dl}$ in mean values between the two breakfast groups, being higher for breakfast eaters. No differences were found, however, in the mean values of either ferritin or $\mathrm{Hb}(13.49 v .13 .82 \mathrm{~g} / \mathrm{dl}$; data for $\mathrm{Hb}$ not shown in table). Similarly, mean values of fasting glucose were $4 \mathrm{mg} / \mathrm{dl}$ higher among breakfast skippers.

Obesity levels, as examined by BMI, did not differ between the two breakfast groups. The mean values of $\mathrm{BF} \%$ and the 'WC and BF\%' variable were slightly higher among breakfast skippers.

Mean levels of LDL-C, HDL-C, TC, TAG and hs-CRP, and mean CDRI values, did not differ significantly by breakfast intake. In addition, physical activity levels were not related to breakfast intake; although median values of physical activity levels (evaluated as pedometer counts) in the two breakfast intake categories differed largely, this difference did not reach statistical significance (median pedometer steps for breakfast-skippers, 18707 ; for breakfast-eaters, 21 493).

Results of the multivariate logistic regression analyses, with breakfast intake frequency as the dependent outcome

Table 1 Descriptive characteristics by breakfast intake status* among eighty-three primary-school children of the Troodos mountainous area in Cyprus, CYFamilies (Cyprus Families) study, 2006/07 school year

\begin{tabular}{|c|c|c|c|c|c|}
\hline & \multicolumn{2}{|c|}{ Breakfast skippers } & \multicolumn{3}{|c|}{ Breakfast eaters } \\
\hline & $\begin{array}{l}\text { Mean, } n \text { or } \\
\text { median }\end{array}$ & sD, \% or IQR & $\begin{array}{l}\text { Mean, } n \text { or } \\
\text { median }\end{array}$ & SD, \% or IQR & $\begin{array}{c}P \\
\text { value }\end{array}$ \\
\hline Age (years), mean and SD & $9 \cdot 10$ & $1 \cdot 71$ & $9 \cdot 28$ & 1.60 & 0.507 \\
\hline \multicolumn{6}{|l|}{ Gender, $n$ and \% } \\
\hline Boys & 42 & 49.4 & 41 & 54.7 & 0.507 \\
\hline Girls & 43 & $50 \cdot 6$ & 34 & $45 \cdot 3$ & \\
\hline BMI $\left(\mathrm{kg} / \mathrm{m}^{2}\right)$, mean and sD & 18.02 & 3.85 & 17.96 & 3.24 & 0.913 \\
\hline WC $(\mathrm{cm})$, mean and SD & 59.71 & $10 \cdot 29$ & 59.50 & 7.93 & 0.887 \\
\hline $\mathrm{BF} \%$, mean and SD & 22.34 & 7.34 & 21.17 & $6 \cdot 41$ & 0.293 \\
\hline $\begin{array}{l}\text { 'WC and BF\%' variable (theoretical range: } 0-2 \text { points (worse)), } \\
\text { mean and SD }\end{array}$ & 0.38 & 0.66 & 0.25 & 0.49 & 0.148 \\
\hline $\begin{array}{l}\text { Modified KIDMED score (theoretical range: }-3 \text { to } 12 \text { points } \\
\text { (best)), mean and SD }\end{array}$ & $6 \cdot 01$ & $2 \cdot 50$ & $7 \cdot 07$ & $2 \cdot 32$ & 0.007 \\
\hline $\mathrm{TC}(\mathrm{mg} / \mathrm{dl})$, mean and SD & $163 \cdot 62$ & 28.65 & $165 \cdot 27$ & $25 \cdot 76$ & 0.786 \\
\hline LDL-C $(\mathrm{mg} / \mathrm{dl})$, mean and SD & $96 \cdot 27$ & 23.14 & 95.89 & 24.55 & 0.943 \\
\hline $\mathrm{HDL}-\mathrm{C}(\mathrm{mg} / \mathrm{dl})$, mean and SD & 51.95 & 10.05 & 54.77 & 10.35 & 0.218 \\
\hline TAG $(\mathrm{mg} / \mathrm{dl})$, mean and SD & 76.97 & 30.89 & 72.68 & 30.64 & 0.533 \\
\hline Fasting glucose $(\mathrm{mg} / \mathrm{dl})$, mean and SD & $90 \cdot 00$ & 6.59 & 86.00 & 6.30 & 0.019 \\
\hline hs-CRP, log transformed (mg/dl), mean and SD & 0.05 & 0.05 & 0.03 & 0.04 & 0.102 \\
\hline Serum $\mathrm{Fe}(\mu \mathrm{g} / \mathrm{dl})$, mean and SD & 69.97 & 27.97 & 82.79 & 29.71 & 0.050 \\
\hline Ferritin (ng/dl), mean and SD & 41.22 & 14.16 & 41.36 & 13.40 & 0.962 \\
\hline CDRI (theoretical range: $0-5$ (worse)), mean and SD & $1 \cdot \overline{16}$ & 1.09 & 1.00 & 1.12 & 0.514 \\
\hline Physical activity level (as pedometer counts), median and IQR & 18707 & $14607-25072$ & 21493 & $17580-29272$ & 0.762 \\
\hline
\end{tabular}

IQR, interquartile range; WC, waist circumference; BF\%, body fat percentage; KIDMED, Mediterranean Diet Quality Index for children and adolescents; TC, total cholesterol; LDL-C, LDL cholesterol; HDL-C, HDL cholesterol; hs-CRP, high sensitivity C-reactive protein; CDRI, cardiovascular disease risk index.

$P$ values obtained by the independent $t$ test for normally distributed variables, by the Mann-Whitney $U$ test for variables with non-normal distribution or by the $\chi^{2}$ test for categorical variables.

*Breakfast skippers, most of the days do not take breakfast; breakfast eaters, most of the days take breakfast. 
Table 2 Results from binary logistic regression analyses evaluating the association between breakfast intake status* (dependent variable) and KIDMED score and obesity indices (independent variables) among eighty-three primary-school children of the Troodos mountainous area in Cyprus, CYFamilies (Cyprus Families) study, 2006/07 school year

\begin{tabular}{|c|c|c|c|c|c|c|c|c|c|c|}
\hline \multirow[b]{2}{*}{ Variables included in the model } & \multicolumn{2}{|c|}{$\begin{array}{c}\text { Model for } \\
\text { Mediterranean } \\
\text { diet adherence }\end{array}$} & \multicolumn{2}{|c|}{$\begin{array}{l}\text { Model for } \\
\text { BF\% }\end{array}$} & \multicolumn{2}{|c|}{$\begin{array}{l}\text { Model for } \\
\text { WC }\end{array}$} & \multicolumn{2}{|c|}{$\begin{array}{l}\text { Model for } \\
\text { BMI }\end{array}$} & \multicolumn{2}{|c|}{$\begin{array}{c}\text { Model for 'WC } \\
\text { and BF\%' } \\
\text { variable }\end{array}$} \\
\hline & OR & $95 \% \mathrm{Cl}$ & OR & $95 \% \mathrm{Cl}$ & OR & $95 \% \mathrm{Cl}$ & OR & $95 \% \mathrm{Cl}$ & OR & $95 \% \mathrm{Cl}$ \\
\hline $\begin{array}{l}\text { Age per } 6 \text { months } \\
\text { Gender }\end{array}$ & 0.94 & $0.66,1.35$ & 0.98 & $0.66,1.45$ & 1.36 & $0 \cdot 85,2 \cdot 18$ & $1 \cdot 24$ & $0.81,1.91$ & 0.98 & $0.67,1.45$ \\
\hline Boys & $1 \cdot 16$ & $0.36,3.79$ & 0.53 & $0 \cdot 13,2 \cdot 20$ & 1.30 & $0.36,4.66$ & 0.93 & $0.25,3.44$ & 1.01 & $0.28,3.62$ \\
\hline Girls & 1.00 & - & 1.00 & - & 1.00 & - & 1.00 & - & 1.00 & - \\
\hline $\begin{array}{l}\text { Modified KIDMED score (range: }-3 \text { to } \\
12 \text { points) per point }\end{array}$ & 1.31 & $1.04,1.66$ & 1.41 & $1.08,1.85$ & 1.42 & $1.09,1.85$ & 1.41 & $1.08,1.84$ & $1 \cdot 39$ & $1.06,1.82$ \\
\hline $\mathrm{BF} \%$ per unit & - & - & 0.86 & $0.77,0.95$ & - & - & - & - & - & - \\
\hline WC per unit & - & - & - & - & 0.89 & $0.81,0.97$ & - & - & - & - \\
\hline BMI per unit & - & - & - & - & - & - & 0.75 & $0.60,0.93$ & - & - \\
\hline 'WC and BF\%' variable (range: 0-2 points) per point & - & - & - & - & - & - & - & - & 0.20 & $0.06,0.69$ \\
\hline Physical activity level (as mean pedometer counts) & 1.00 & $1.00,1.00$ & 1.00 & $1.00,1.00$ & 1.00 & $1.00,1.00$ & 1.00 & $1.00,1.00$ & 1.00 & $1.00,1.00$ \\
\hline
\end{tabular}

KIDMED, Mediterranean Diet Quality Index for children and adolescents; BF\%, body fat percentage; WC, waist circumference.

${ }^{*}$ Regular breakfast intake (no v. yes).

and $\mathrm{BMI}, \mathrm{WC}, \mathrm{BF} \%$, 'WC and $\mathrm{BF} \%$ ' variable and modified KIDMED score as independent variables, are presented in Table 2. After considering potential confounders, significant inverse associations were observed between breakfast intake and $\mathrm{BMI}, \mathrm{WC}, \mathrm{BF} \%$ and the ' $\mathrm{WC}$ and $\mathrm{BF} \%$ ' variable, whereas a positive association was revealed between breakfast intake and adherence to the Mediterranean diet (assessed via the KIDMED score). Specifically, breakfast eaters were on average $40 \%$ more likely to have a modified KIDMED score higher by one point, after accounting for obesity levels and other confounders (e.g. model for $\mathrm{BF} \%$ : $\mathrm{OR}=1.41 ; 95 \% \mathrm{CI} 1.08,1 \cdot 85)$. Breakfast skippers, on the other hand, were $14 \%$ more likely to exhibit a one unit increase in $\mathrm{BF} \%(\mathrm{OR}=0.86 ; 95 \% \mathrm{CI} 0.77,0.95)$. Of same direction and similar magnitude was the relationship of BMI and WC to breakfast intake, and this relationship was significantly strengthened when combining $\mathrm{BF} \%$ and WC into one categorical variable $(\mathrm{OR}=0 \cdot 20 ; 95 \% \mathrm{CI}$ 0.06, 0.69).

The significant association between fasting glucose levels and breakfast intake observed in univariate descriptive analyses disappeared when a multivariate assessment was performed (results not shown here). With regard to serum $\mathrm{Fe}$, it is of note that the relationship disappeared only after considering physical activity levels; when only age, gender and diet quality were used as covariates, children who regularly ate breakfast were $80 \%$ less likely to have serum Fe levels lower than $65 \mu \mathrm{g} / \mathrm{dl}(\mathrm{OR}=0 \cdot 20$; $95 \%$ CI 0.07, 0.50; data not shown here).

\section{Discussion}

We have presented the results of the CYFamilies study conducted among Cypriot primary-school children living in the mountainous area of Cyprus, examining the relationship between breakfast intake and lifestyle attributes.
The findings provide evidence that, after considering potential confounders, regular breakfast eaters were more likely to have higher adherence to the Mediterranean diet and lower BF\%, BMI, WC and 'WC and BF\%' variable than breakfast skippers. It is of note that serum Fe was positively associated with breakfast intake when controlling for age, gender and diet quality, but the association attenuated when physical activity was considered. Furthermore, regular breakfast intake was negatively associated with fasting glucose, but in descriptive analyses only.

\section{Diet quality}

The relationship between aspects of diet quality and breakfast intake is well established ${ }^{(1,2,9)}$; studies on the association of breakfast intake with overall diet quality, however, are rather limited. In our study, a dietary index based on the Mediterranean diet prototype was used in order to assess the overall quality of diet. Our results are in line with a number of other studies that have also assessed diet quality via various holistic dietary indices (KIDMED index $^{(4)}$, Modified Diet Quality Index International ${ }^{(5)}$, Diet Quality Index International ${ }^{(10)}$ and Healthy Eating Index- $\mathrm{C}^{(11)}$ ) and showed that breakfast intake is associated with overall diet quality. To the best of our knowledge, the present study is the first one to evaluate the association of breakfast intake with Mediterranean diet adherence assessed by a modified KIDMED index, i.e. after omitting its breakfast component to exclude any bias in results. Adoption of the habit of taking a morning meal is regarded as an essential behaviour modification towards establishing a healthful diet and is also a target outcome in large public health initiatives among children ${ }^{(26,27)}$. In the present study an association of serum Fe with children's breakfast intake was detected, revealing a difference in the mean values of serum Fe between breakfast eaters and breakfast skippers as high as $10 \mu \mathrm{g} / \mathrm{dl}$. However, this 
association disappeared after considering physical activity and, moreover, none of the other two Fe status indicators (ferritin and $\mathrm{Hb}$ ) was significantly associated with breakfast intake, even in univariate descriptive analyses. Breakfast has been reported to be an important contributor to vitamin and mineral intakes (including $\mathrm{Fe}$ ) in children ${ }^{(12,44)}$ and improved Fe status indicators are therefore expected, mainly because of the Fe-fortified ready-to-eat breakfast cereals commonly eaten at breakfast. Physical activity was found to be an important confounder, however, and appears to be a factor that mediates the relationship between serum Fe and breakfast. Moreover, it should also be recognized that serum $\mathrm{Fe}$ is an insensitive indicator of total Fe stores and may be extremely variable from day to day. Another explanation for the inability of the present study to demonstrate a stable association of any of the indicators of body Fe status with breakfast intake may be the small sample size. In any case, the relationship of breakfast intake with levels of nutritional biomarkers remains under-searched and deserves further study.

\section{Obesity}

Despite our expectations, examination of direct associations detected no significant relationships between breakfast intake and $\mathrm{BMI}, \mathrm{WC}, \mathrm{BF} \%$ and the 'WC and $\mathrm{BF} \%$ ' variable (Table 1). (Being not a statistically significant difference, mean BF\% was, however, one point higher among breakfast skippers.) In contrast, when the above variables were entered into multivariate logistic regression analysis, statistically significant relationships with breakfast intake emerged. Notably, when the composite variable 'WC and BF\%' was entered in the model, a significant synergistic effect between the two variables became apparent, as revealed by the almost $60 \%$ 'improvement' in the mean odds ratio between breakfast intake and the 'WC and BF\%' variable compared with models where $\mathrm{BMI}$, WC or $\mathrm{BF} \%$ was used alone. Our findings are in line with the majority of previous studies, which are summarized in three reviews ${ }^{(2,9,21)}$. It is of note that while earlier reviews included cross-sectional studies mainly, the most recent and systematic review of the 2010 American Dietary Guidelines Committee excluded cross-sectional studies and, taking into account prospective and interventional data, concluded there is good evidence that children who do not eat breakfast are at increased risk for being overweight and obese; the evidence was stronger for adolescents ${ }^{(9)}$.

An advantage of our study is the use of four adiposity markers, including $\mathrm{BF} \%$, which is regarded as a more accurate method to assess obesity. A number of possible mechanisms may lie behind the inverse relationship of obesity with breakfast eating: (i) regular breakfast eaters generally have a better health profile and healthy lifestyle, including increased physical activity levels ${ }^{(18,45-47)}$, factors that have been associated with lower obesity levels; (ii) breakfast skippers have been found to have lower diet quality, increased consumption of high-energy/low-nutrient-density foods $^{(48)}$ and higher energy intakes ${ }^{(13,17,46)}$, whereas regular breakfast intake is associated with consistent energy intake ${ }^{(48)}$; (iii) breakfast omission is associated with overconsumption of food and energy intake later in the day $^{(16,17,46)}$, presumably because of increased hunger ${ }^{(49)}$; and (iv) large fluctuations in energy intake may lead to hyperinsulinaemia, which in turn may negatively influence metabolic rate ${ }^{(50,51)}$ and may promote fat storage.

\section{Cardiovascular risk}

Fasting glucose levels were higher in children who were classified as breakfast skippers, compared with their counterparts, but only in descriptive analyses. None of the other cardiovascular risk markers (blood lipids and CDRI) was significantly associated with breakfast intake in either descriptive or multivariate analyses, despite the fact that the lipid profiles of breakfast skippers were slightly worse. The inability to reveal a potentially significant relationship may be due to the low number of participants or was mediated through obesity (at least partly), as others have reported $^{(52,53)}$. Indeed, when an obesity marker was incorporated into the CDRI, similarly to previous studies in children $^{(50,54)}$, breakfast intake became significantly associated with CDRI, even after controlling for confounders (data not shown). Generally, literature in this area is limited but available information from experimental, prospective and nationwide studies in adults ${ }^{(49,51,52,55,56)}$, as well as some studies in children ${ }^{(19,53,57)}$, provide evidence that omission of breakfast may impair serum lipid, postprandial insulin and glucose homeostasis. Furthermore, the importance of breakfast intake to cardiovascular health has been recently highlighted by findings in a prospective cohort of almost 27000 healthy US men, indicating that men who skipped breakfast had a $27 \%$ higher risk of cardiovascular heart disease ${ }^{(58)}$.

\section{Physical activity levels}

Although in our study the observed differences in physical activity levels, as shown in Table 1 , did not reach statistical significance, it is of note that the difference in median values between the two breakfast intake categories was about 2500 steps/d. The relationship of breakfast intake with physical activity is generally less well studied than its relationship with diet quality and obesity. However, there are a number of studies that report a positive relationship of breakfast intake with physical activity levels ${ }^{(44,59)}$, suggesting that regular breakfast eaters may be people who generally are more concerned about their health and more likely to pursue healthy lifestyles.

\section{Strengths and limitations}

Among the strengths of the present study is the use of four adiposity markers, including $\mathrm{BF} \%$, which were all significantly associated with breakfast intake in multivariate analysis. Second, the study is the first in children which examined the association of breakfast intake with 
Mediterranean diet adherence (after excluding the component of breakfast intake from the Mediterranean diet quality index for children, KIDMED). Third, even though the differences observed among the two categories of breakfast eaters in serum $\mathrm{Fe}$, fasting glucose (data not shown) and physical activity levels did not reach statistical significance in multivariate analyses, it may be of practical significance and provides evidence of the favourable effects of breakfast intake on general health.

The present study has certain limitations which should be taken into account. First, it was designed as a crosssectional study. Therefore, no conclusions can be made about plausible causes, but rather indications can be extracted. Second, there was only single measurement of blood tests which could have caused a misclassification of cases and thus influenced the results of the examined associations. However, none of the other studies in children, that had blood tests, had multiple blood measurements. Moreover, the sample size may not have been sufficiently powered to detect some associations. Nevertheless, we feel that our findings are in accordance with similar larger studies and, considering the scarcity of relevant data especially in Mediterranean and European populations, they may contribute in advancing knowledge and understanding of the importance of breakfast to children's health.

\section{Conclusions and implications}

In conclusion, the present study demonstrates that Cypriot children who are regular breakfast consumers have higher adherence to the Mediterranean diet, lower BMI, WC and $\mathrm{BF} \%$, as shown by multivariate analyses, as well as higher serum $\mathrm{Fe}$ concentrations and lower fasting glucose, as shown by descriptive analyses. Public health professionals, educators and parents should prioritize on actions that will motivate children to regularly take breakfast.

\section{Acknowledgements}

Acknowledgements: The authors would like to thank the Ministry of Health and the State Hospital of Kyperounda for enabling the conduction of the study. Financial support: The study was supported by Cyprus Research Promotion Foundation and Vouros Chemicals' Company. Cyprus Research Promotion Foundation and Vouros Chemicals' Company had no role in the design, analysis or writing of this article. Conflict of interest: None. Authorship: The contributions of the authors were as follows. C.L. was responsible for the study design, obtaining the funding, the collection of data, the data analysis and the preparation of the manuscript; A.-L.M. contributed to the presentation and interpretation of the results and critically reviewed the paper. Both authors contributed to the final version of the manuscript. Ethics of human subject participation: The research design was approved by the
Ministries of Education and Culture (Department of Primary Education) and Health, as Cypriot law provides for studies that are performed in the school environment during formal school hours.

\section{References}

1. Ruxton $\mathrm{CH}$ \& Kirk TR (1997) Breakfast: a review of associations with measures of dietary intake, physiology, and biochemistry. Br J Nutr 78, 199-213.

2. Rampersaud GC, Pereira MA, Girand BL et al. (2005) Breakfast habits, nutritional status, body weight, and academic performance in children and adolescents. $\mathrm{J} \mathrm{Am}$ Diet Assoc 105, 743-760.

3. Hoyland A, Dye L \& Lawton CL (2009) A systematic review of the effect of breakfast on the cognitive performance of children and adolescents. Nutr Res Rev 22, 220-243.

4. Kontogianni MD, Farmaki AE, Vidra N et al. (2010) Associations between lifestyle patterns and body mass index in a sample of Greek children and adolescents. $J$ Am Diet Assoc 110, 215-221.

5. Mariscal-Arcas M, Rivas A, Velasco J et al. (2009) Evaluation of the Mediterranean Diet Quality Index (KIDMED) in children and adolescents in Southern Spain. Public Health Nutr 12, 1408-1412.

6. Affenito SG, Thompson DR, Barton BA et al. (2005) Breakfast consumption by African-American and white adolescent girls correlates positively with calcium and fiber intake and negatively with body mass index. J Am Diet Assoc 105, 938-945.

7. Radzikowski J \& Gale S (1984) The national evaluation of school nutrition programs: conclusions. Am J Clin Nutr $\mathbf{4 0}$, 454-461.

8. Hanes S, Vermeersch J \& Gale S (1984) The national evaluation of school nutrition programs: program impact on dietary intake. Am J Clin Nutr 40, 390-413.

9. US Department of Agriculture \& US Department of Health and Human Services (2010) Report of the Dietary Guidelines Advisory Committee on the Dietary Guidelines for Americans. Washington, DC: US Government Printing Office.

10. Veugelers PJ, Fitzgerald AL \& Johnston E (2005) Dietary intake and risk factors for poor diet quality among children in Nova Scotia. Can J Public Health 96, 212-216.

11. Woodruff SJ, Hanning RM, Lambraki I et al. (2008) Healthy Eating Index-C is compromised among adolescents with body weight concerns, weight loss dieting, and meal skipping. Body Image 5, 404-408.

12. Nicklas TA, O'Neil CE \& Berenson GS (1998) Nutrient contribution of breakfast, secular trends, and the role of ready-to-eat cereals: a review of data from the Bogalusa Heart Study. Am J Clin Nutr 67, issue 4, 757S-763S.

13. Nicklas TA, Reger C, Myers L et al. (2000) Breakfast consumption with and without vitamin-mineral supplement use favorably impacts daily nutrient intake of ninth-grade students. J Adolesc Health 27, 314-321.

14. Ortega RM, Requejo AM, Lopez-Sobaler AM et al. (1998) The importance of breakfast in meeting daily recommended calcium intake in a group of schoolchildren. Am J Coll Nutr 17, 19-24.

15. Sampson AE, Dixit S, Meyers AF et al. (1995) The nutritional impact of breakfast consumption on the diets of inner-city African-American elementary school children. J Natl Med Assoc 87, 195-202.

16. Moreno LA \& Rodríguez G (2007) Dietary risk factors for development of childhood obesity. Curr Opin Clin Nutr Metab Care 10, 336-341.

17. Dubois L, Girard M, Potvin Kent M et al. (2009) Breakfast skipping is associated with differences in meal patterns, 
macronutrient intakes and overweight among pre-school children. Public Health Nutr 12, 19-28.

18. Keski-Rahkonen A, Kaprio J, Rissanen A et al. (2003) Breakfast skipping and health-compromising behaviors in adolescents and adults. Eur J Clin Nutr 57, 842-853.

19. Resnicow K (1991) The relationship between breakfast habits and plasma cholesterol levels in schoolchildren. $J$ Sch Health 61, 81-85.

20. Firore H, Travis S, Whalen A et al. (2006) Potentially protective factors associated with healthful body mass index in adolescents with obese and nonobese parents: a secondary data analysis of the Third National Health and Nutrition Examination Survey, 1988-1994. J Am Diet Assoc 106, 55-64.

21. Szajewska H \& Ruszczynski M (2010) Systematic review demonstrating that breakfast consumption influences body weight outcomes in children and adolescents in Europe. Crit Rev Food Sci Nutr 50, 113-119.

22. Utter J, Scragg R, Ni Mhurchu C et al. (2007) At-home breakfast consumption among New Zealand children: associations with body mass index and related nutrition behaviors. J Am Diet Assoc 107, 570-576.

23. Croezen S, Visscher TL, Ter Bogt NC et al. (2009) Skipping breakfast, alcohol consumption and physical inactivity as risk factors for overweight and obesity in adolescents: results of the E-MOVO project. Eur J Clin Nutr 63, 405-412.

24. Niemeier HM, Raynor HA, Lloyd-Richardson EE et al. (2006) Fast food consumption and breakfast skipping: predictors of weight gain from adolescence to adulthood in a nationally representative sample. J Adolesc Health 39, 842-849.

25. Barlow SE \& Expert Committee (2007) Expert committee recommendations regarding the prevention, assessment, and treatment of child and adolescent overweight and obesity: summary report. Pediatrics 120, Suppl. 4, S164-S192.

26. Hirschman J \& Chriqui JF (2013) School food and nutrition policy, monitoring and evaluation in the USA. Public Health Nutr 16, 982-988.

27. Moore L, Moore GF, Tapper K et al. (2007) Free breakfasts in schools: design and conduct of a cluster randomised controlled trial of the Primary School Free Breakfast Initiative in Wales [ISRCTN18336527]. BMC Public Health 7, 258.

28. Breakfast is Best campaign (2013) Home page. http://www. breakfastisbest.eu/ (accessed July 2013).

29. Grivetti LE (1997) Morning meals. North American and Mediterranean breakfast patterns. Part 3. Mediterranean patterns and summary. Nutr Today 32, 164-171.

30. Yiagkou V, Demetriou D, Lazarou C et al. (2011) A king with rugs. Mealhabits and traditional products on Cyprus. Hellen J Nutr Diet 2, 87-95 (in Greek).

31. Lazarou C, Panagiotakos DB, Chrysohoou C et al. (2010) C-reactive protein levels are associated with adiposity in mountainous Cypriot children: The CyFamilies Study. Clin Nutr 29, 779-783.

32. Lazarou C, Panagiotakos DB, Kouta C et al. (2009) Dietary and other lifestyle characteristics of Cypriot children: results from the nationwide CYKIDS study. BMC Public Health 9, 147.

33. Matalas A-L, Lazarou C \& Panagiotakos DB (2012) Meal habits of Cypriot schoolchildren: associations with socioeconomic factors. In Time for Food: Everyday Meals and Changing Food Habits, pp. 237-246 [P Lysaght, editor]. Turku: Åbo Academi University Press.

34. Lazarou C, Karaolis M, Matalas AL et al. (2012) Dietary patterns analysis using data mining method. An application to data from the CYKIDS study. Comput Methods Programs Biomed 108, 706-714.

35. Serra-Majem L, Ribas L, García A et al. (2003) Nutrient adequacy and Mediterranean Diet in Spanish school children and adolescents. Eur J Clin Nutr 57, Suppl. 1, S35-S39.
36. Loucaides CA, Chedzoy SM \& Bennett N (2004) Differences in physical activity levels between urban and rural school children in Cyprus. Health Educ Res 19, 138-147.

37. Heymsfield SB, Lohman TG, Wang Z et al. (editors) (2005) Human Body Composition, 2nd ed. Champaign, IL: Human Kinetics.

38. Cole TJ, Bellizzi MC, Flegal KM et al. (2000) Establishing a standard definition for child overweight and obesity worldwide: international survey. BMJ 320, 1240-1243.

39. McCarthy HD, Cole TJ, Fry T et al. (2006) Body fat reference curves for children. Int J Obes (Lond) 30, 598-602.

40. Savva SC, Tornaritis M, Savva ME et al. (2000) Waist circumference and waist-to-height ratio are better predictors of cardiovascular disease risk factors in children than body mass index. Int J Obes Relat Metab Disord 24, 1453-1458.

41. Lazarou C \& Soteriades ES (2010) Children's physical activity, TV watching and obesity in Cyprus: the CYKIDS study. Eur J Public Health 20, 70-77.

42. American Heart Association (n.d.) Children and Cholesterol. http://www.heart.org/HEARTORG/Conditions/Cholesterol/ UnderstandYourRiskforHighCholesterol/Children-andCholesterol_UCM_305567_Article.jsp (accessed July 2013).

43. Pearson TA, Mensah GA, Alexander RW et al. Centers for Disease Control and Prevention; American Heart Association (2003) Markers of inflammation and cardiovascular disease: application to clinical and public health practice: a statement for healthcare professionals from the Centers for Disease Control and Prevention and the American Heart Association. Circulation 107, 499-511.

44. Albertson AM, Anderson GH, Crockett SJ et al. (2003) Ready-to-eat cereal consumption: its relationship with BMI and nutrient intake of children aged 4 to 12 years. J Am Diet Assoc 103, 1613-1619.

45. Vereecken C, Dupuy M, Rasmussen M et al. HBSC Eating \& Dieting Focus Group (2009) Breakfast consumption and its socio-demographic and lifestyle correlates in schoolchildren in 41 countries participating in the HBSC study. Int J Public Health 54, Suppl. 2, 180-190.

46. Sjoberg A, Hallberg L, Hoglund D et al. (2003) Meal pattern, food choice, nutrient intake, and lifestyle factors in the Goteborg Adolescence Study. Eur J Clin Nutr 57, 1569-1578.

47. Sandercock GR, Voss C \& Dye L (2010) Associations between habitual school-day breakfast consumption, body mass index, physical activity and cardiorespiratory fitness in English schoolchildren. Eur J Clin Nutr 64, 1086-1092.

48. Timlin MT \& Pereira MA (2007) Breakfast frequency and quality in the etiology of adult obesity and chronic diseases. Nutr Rev 65, 268-281.

49. Astbury NM, Taylor MA \& Macdonald IA (2011) Breakfast consumption affects appetite, energy intake, and the metabolic and endocrine responses to foods consumed later in the day in male habitual breakfast eaters. J Nutr 141, 1381-1389.

50. Sesé MA, Jiménez-Pavón D, Gilbert CC et al.; HELENA Study Group (2012) Eating behaviour, insulin resistance and cluster of metabolic risk factors in European adolescents. The HELENA study. Appetite 59, 140-147.

51. Farshchi HR, Taylor MA \& Macdonald IA (2005) Deleterious effects of omitting breakfast on insulin sensitivity and fasting lipid profiles in healthy lean women. Am J Clin Nutr 81, 388-396.

52. Mekary RA, Giovannucci E, Cahill L et al. (2013) Eating patterns and type 2 diabetes risk in older women: breakfast consumption and eating frequency. Am J Clin Nutr 98, 436-443.

53. Smith KJ, Gall SL, McNaughton SA et al. (2010) Skipping breakfast: longitudinal associations with cardiometabolic risk factors in the Childhood Determinants of Adult Health Study. Am J Clin Nutr 92, 1316-1325.

54. Bel-Serrat S, Mouratidou T, Börnhorst C et al. (2013) Food consumption and cardiovascular risk factors in 
European children: the IDEFICS study. Pediatr Obes $\mathbf{8}$, 225-236.

55. Deshmukh-Taskar P, Nicklas TA, Radcliffe JD et al. (2013) The relationship of breakfast skipping and type of breakfast consumed with overweight/obesity, abdominal obesity, other cardiometabolic risk factors and the metabolic syndrome in young adults. The National Health and Nutrition Examination Survey (NHANES): 1999-2006. Public Health Nutr 16, 2073-2082.

56. di Giuseppe R, Di Castelnuovo A, Melegari C et al.; Moli-sani Project Investigators (2012) Typical breakfast food consumption and risk factors for cardiovascular disease in a large sample of Italian adults. Nutr Metab Cardiovasc Dis 22, 347-354.
57. Hallström L, Labayen I, Ruiz JR et al.; HELENA Study Group (2013) Breakfast consumption and CVD risk factors in European adolescents: the HELENA (Healthy Lifestyle in Europe by Nutrition in Adolescence) Study. Public Health Nutr 16, 1296-1305.

58. Cahill LE, Chiuve SE, Mekary RA et al. (2013) Prospective study of breakfast eating and incident coronary heart disease in a cohort of male US health professionals. Circulation 128, 337-343.

59. Godin G, Anderson D, Lambert LD et al. (2005) Identifying factors associated with regular physical activity in leisure time among Canadian adolescents. Am J Health Promot 20, $20-27$. 